\title{
Numerical Simulation and Implementation of FBMC System with Low PAPR for Efficient 5G Applications
}

\author{
Dr. Sunil Kumar Gupta \\ Professor, EEE, School of Engineering \& Technology, Poornima University, Jaipur, India \\ sunil.gupta@poornima.edu.in
}

\author{
Article Info \\ Page Number: $94-98$ \\ Publication Issue: \\ Vol 71 No. 1 (2022)
}

\begin{abstract}
Recently, FBMC (Filter Bank Multi-Transporter) has emerged as an alternative to orthogonal frequencies Division Multiplexing (OFDM) $(\mathrm{OFDM})$. As part of the FBMC strategy, wider ranges of frequencies are now within reach, as well as lower out-of-band (OOB) outflows. Comparatively speaking, The Peak-to-average Power Ratio (PAPR) in OFDM frameworks is still high for FBMC, so this isn't surprising. The FBMC framework has been given some OFDM-based PAPR reduction innovation.. PAPR advancements are reduced in this paper OFDM frameworks employ phase modification (PR) and modified PR (MPR) algorithms as a foundation for their proposed FBMC framework. Simply integrate DFT (Discrete Fourier Transform) Spread range and FBMCOQAM (Filter Bank Multi-Transporter) into SC-FDMA (Single Bearer Recurrence Division Multiple Entrance). As long as you keep the quadrature sufficiency tweak in check, PAPR (peak normal power proportion), which was formerly negligible, is now much less. Taking advantage of DFT's single-bearer impact, unique approaches can be taken. There should be no problems with the quadrature phase channel in terms of the IQ coefficients for each subcarrier. We use FBMC waveforms to generate DFT augmentation and ITSM conditions, and then select the one with the lowest pinnacle power as a means of further increasing the amount decreased PAPR. Regardless of the number of candidates, the basic count portion, for example, DFT Share with IDFT, only executes once, unlike the typical SI (side data) based PAPR reduction Programs.
\end{abstract}

Keywords: - FBMC,PAPR, OFDM, PSNR, BER,DFT.
Article History

Article Received: 18 November 2021

Revised: 01 December 2021

Accepted: 15 December 2021

Publication: 27 January 2022

\section{INTRODUCTION}

"Greater, quicker, higher? The craving for broadband has obviously powered the advancement of portable cell systems. Then again, the effective sending of executioner applications in the previous 20 years has majorly affected the business sectors too: First and premier the requirement for un-fastened communication and in this way remote constant voice correspondence has ruled the accomplishment of cordless telephones, trailed by original (1G) of cell interchanges. Before long, joined in the subsequent age (2G), two-way paging executed by short message administration (SMS) content informing turned into the second executioner application. With the accomplishment of remote neighborhood (WLAN) innovation (for example IEEE 802.11), Internet perusing, and the across the board showcase appropriation of smart phones, information availability wound up intriguing for anybody, opening up the open 
door for making a business opportunity for the third executioner application in third era (3G): remote information network. The consistent following stage has been the shrinkage of the workstation, blending it with the phone into the present cell phones, and offering high transfer speed access to remote clients with the world's data readily available all over and unfailingly. This is the situation of the present fourth era (4G), alleged Long Term Evolution - Advanced (LTE-A). Cell phones are, without a doubt, in the focal point of administration designs for future versatile access. Presently, is there an amazing application for 5G seemingly within easy reach?

Essential research for $5 \mathrm{G}$ is well under way. The principle drivers are:

-Internet of Things (IoT): The IoT will surely assume a key job yet plans of action have not begun off yet. The fundamental test is the versatility issue with more than, state, 100k machine-type correspondence (MTC) hubs in a cell under the premises of ease (underneath $10 \$$ per radio module) and life time (more noteworthy than 10 years). The IoT could change the manner in which we consider the To be as a human-to-human interface towards a progressively broad machine-to-machine stage.

-Gigabit Wireless Connectivity: For instance, clients may demand speedy downloads (for example from a remote information stand) of 3D spilling substance with information rates in the request of $\sim 100 \mathrm{Mbit} / \mathrm{s}$. Accordingly, download times are relied upon to be multiple times quicker, in this manner, in the request of $\sim 10 \mathrm{Gbit} / \mathrm{s}$. Gigabit remote availability is additionally expected in huge group social events with potentially intuitively associated gadgets (cell phones, tablets, and so forth.).

- Tactile Internet: It involves a huge measure of continuous applications with very low inertness prerequisites. Spurred by the material feeling of the human body which can recognize latencies of the request of $1 \mathrm{~ms}$ exactness, $5 \mathrm{G}$ would then be able to be connected for directing and control situations inferring a troublesome change from the present substance driven interchanges; mainstream thoughts go from virtual overlay of setting data on a showcase, through mechanical autonomy and medicinal services to vehicle security and brilliant city applications. A $1 \mathrm{~ms}$ roundtrip time for a run of the mill material association requires a period spending

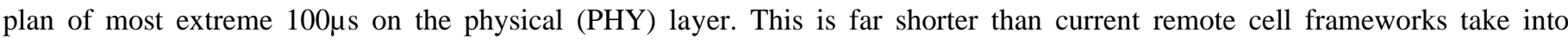
consideration, missing the objective by almost two requests of extent and most likely some more.

From a specialized viewpoint it is by all accounts most extreme testing to give uniform administration experience to clients under the premises of heterogeneous systems administration or future little cell situations. Not exclusively should the system administrators be solid and steady to assume the test of an a lot higher per-client rate and expanding in general required data transfer capacity yet additionally to acknowledge administration separation with altogether different (for all intents and purposes repudiating) application prerequisites. Thusly, the radio access must be adaptable, versatile, content mindful, powerful, solid and productive as far as vitality and range. All things considered, with the constraints of current $4 \mathrm{G}$ framework, this will put further weight on the basic worth chains on which the administrators depend so as to make up for venture costs for future client administrations. Consequently, there is an unmistakable inspiration for an inventive and to a limited extent problematic re-plan of the Physical layer.

\section{NEED OF NEW WAVEFORM}

The fundamental speculation of this article is that, explicitly, the hidden structure standards - synchronism and orthogonality-of the PHY layer of the present LTE-A radio access system establish a noteworthy snag for the imagined administration design. Orthogonality implies that if there should arise an occurrence of flawless synchronized transmission no crosstalk happens. In addition, synchronicity implies that the senders work with a typical clock for their preparing. OFDM tweak keeps the subcarrier waveforms orthogonal even after the channel, gave the DFT window can be appropriately balanced by reasonable synchronization component, which is then close ideal preparing in a solitary cell. Be that as it may, when the orthogonality is decimated, for example by irregular access or multi-cell task, the mutilation gathers without limits in OFDM. This is expected to the supposed imitating Dirichlet portion $\sin (\mathrm{Nx}) / \sin (\mathrm{x})$ of OFDM which rapidly approaches the $\sin (\mathrm{x}) / \mathrm{x}$ piece for enormous $\mathrm{N}$ where $\mathrm{N}$ is the quantity of subcarriers. Consequently, we trust it is smarter to surrender orthogonality out and out and control the debilitations give us a chance to talk about a few captivating models.

\section{FBMC (FILTER BANK MULTI-CARRIER)}

Modulation with the FBMC scheme can cover a wide variety of frequencies and pitches. Scheme utilising a number of different carriers Subchannel modulation is carried out by IFFT - a technique similar to OFDM - before being filtered through a custom-built prototype filter for each subchannel. Has a variety of filters available. For example, it can be used with FBMC. The

In terms of the transmitted signal's spectral properties, this filter has a significant impact. The FBMC transmitter block diagram is introduced in the first paragraph of this section. Spectrum metrics as well as descriptive statistics Analyze the signal modulation. Filter prototype using FBMC modulation to modulate the pulse width. The subcarriers receive the answer of $\mathrm{p} 0$ from the main

Vol. 71 No. 1 (2022)

http://philstat.org.ph 
carrier. The Nyquist criterion is met by these filters. Because The signal's spectral efficiency will be higher than that of an OFDM signal. The FBMC filter bank has been added. Systems such as cognitive radio and opportunity dynamics are attracting research interest. Access to the spectrum. The most likely scenario is that Instead of using orthogonal frequency division multiplexing, this technique can be used (OFDM). As an alternative to OFDM, FBMC was presented [1] to increase spectral efficiency and low radiation with $(\mathrm{OOB})$. A reliable local source of waveforms is available. Both domains can benefit from flexible use of resources and assistance Increase the amount of calculation required. Complexity, on the other hand, By implementing a multi-phase implementation, this can be considerably decreased [2]. But while OFDM has its advantages, the FBMC system does have a number of serious flaws. The signal's peak-to-average power ratio (PAPR). PAPR can't apply reduction techniques for OFDM systems in the FBMC system because of the overlapping structure of the FBMC signals. Reducing PAPR in OFDM systems is becoming more commonplace (eg [3], [4]) The FBMC system There are a number of different research areas. Reduce the FBMC system's PAPR from [5] to [8]. On the basis of active constellation growth, PAPR FBMC technology can be reduced. [9] introduces the FBMC protocol. Due to significant PAPR reduction system complexity, both PTS and SLM may require more information to be shared. In [8], a PAPR reduction method is utilised.

\section{SYSTEM DESIGN}

The parallel data is encoded using a convolutional encoder in the transmitter, and then interleaved subsequently. For each symbol $\mathrm{X}, \mathrm{M}$ bits are assigned using the complex A regulatory letter set A. The legitimate (R) and imaginative (I) parts of the unexpected regulatory symbol X are sent with a period balance of nearly the entire symbol span when counterbalance QAM modification is used. In the end, before transmission, the symbols are covered so that the receiver can isolate them from the rest of the signal. In FBMC frameworks, orthogonality of the subcarriers is maintained by not using CP. Communication of the FBMC baseband signal $\mathrm{s}[\mathrm{n}]$ depends on the mind-boggling balance symbol $\mathrm{Xm}[\mathrm{k}]$ at the subcarrier $\mathrm{k}$.

$$
[n]=\sum_{m=-\infty}^{\infty} \sum_{k=0}^{N-1}\left(\theta_{k} \Re X_{m}[k] p o[n-m N]+\theta_{K}+1 \Im\left\{X_{m}[k]\right\} p o\left[n-m N-\frac{n}{2}\right] e^{j k(n-m N) \frac{2 \pi}{n}}\right)
$$

Figure 3 depicts the block diagram of an FBMC transmitter. Using the adjust letters A, the encoded bitstream $\mathrm{b}$ is translated to a coded bitstream $\mathrm{c}$ where the bits are assigned to complex symbols X. An IFFT and a polyphase decay of the altered model filters for the real and fantastical sections finally make it possible to compute Equation (1) successfully. Both yield signals are then timeawed and incorporated at this stage. A thorough understanding of the measuring qualities of the sent signal is required to plan the simple circuits of the handset chain.

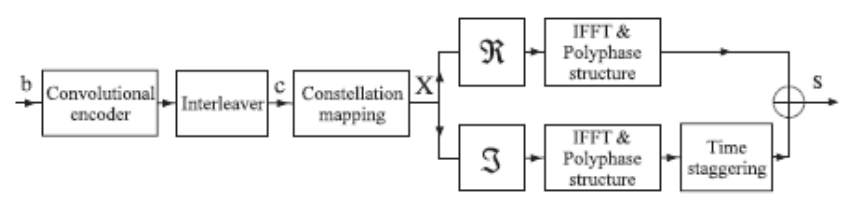

Fig 3.Block Diagram of FBMC Scheme

To design the phone's simple circuits in a way that maximises their efficiency.Chain a thorough understanding of the signal's measuring qualities must be put together. These kinds of tests are particularly important in the structure of power amplifiers that need to perform efficiently. The PAPR is a fundamental step in illustrating the elements of the transmission signal s[n].

as

$$
\gamma_{1}=\frac{\max \left\{|s[n]|^{2}\right\}}{E\left\{|s[n]|^{2}\right\}}
$$

where $|s[n]|$ is the amplitude of the transmission signal and $E\{$.$\} is the expectation value. The PAPR in \mathrm{dB}$ is defined as:

$$
\operatorname{PAPR}(s[n]) d B=10 \log _{-} 10\left(\gamma_{1}\right)
$$




\section{SimUlation \& RESUlt}

As a part of this project, we've created a There were four pilots and 24 data symbols on each RB that represented 14 subcarriers over two OFDM symbols in time for the Wimax standard. An option we've investigated is a $10-\mathrm{MHz}$ system with up to 60 radio frequency (RB) components altogether. Data subcarriers with QPSK modulation plus 92 guard subcarriers at either end of the band make up the bulk of an OFDM block, which is typically 1024 bytes in size. There are one, two, or four transmit antennas in a multiantenna system.

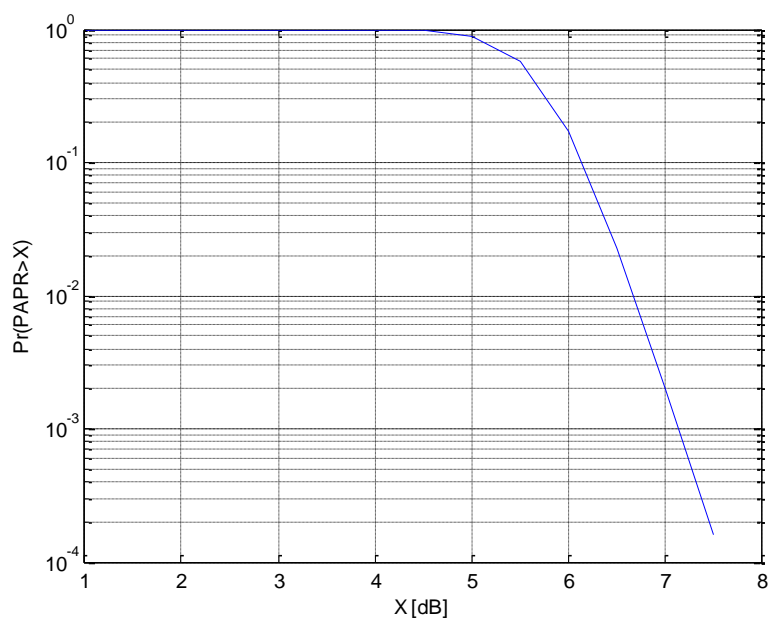

Fig 4. PAPR of FBMC Scheme

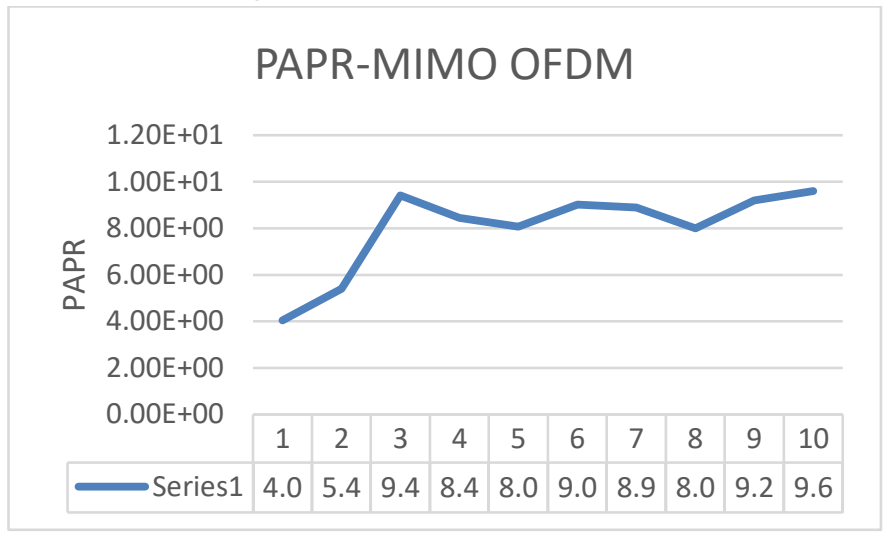

Fig 4. PAPR-OFDM

The CCDF curves are constructed using 10,000 random OFDM blocks. The beam forming matrices are selected as the right singular vectors of these channel matrices, and a random complex fading channel is constructed for each block. A comparison between MIMO-OFDM and DFT-spread FBMC with the earlier DFT-spread method based on PAPR results is first made. For OQPSK with $\mathrm{N}=128$, OQPSK with $\mathrm{N}=64$ and 16 OQAM with $\mathrm{N}=128$, the PAPR's CCDF curves are shown in Fig. 7. The DFT Spread-PAPR FBMC's CCDF curves exhibit superior results than the MIMO-OFDM system.

\section{CONCLUSION}

Here, we propose and demonstrate the superior performance of a low PAPR FBMC technique compared to the already available PAPR reduction schemes.Overhead in terms of gain, computational complexity, and metrication. MIMO OFDM System was first developed for analysis. It was determined that FBMC performs better in terms of PAPR than the FBMC scheme, making it an excellent contender for use as a modulation scheme in the $5 \mathrm{G}$ network..

\section{REFERENCES}

[1] T. Ihalainen, T. Hidalgo Stitz, M. Rinne, and M. Renfors, "Channel equalization in filter bank based multicarrier modulation for wireless communications," EURASIP J. Adv. Signal Process., vol. 2007 
[2] J. Vihriala, N. Ermolova, E. Lahetkangas, O. Tirkkonen, and K. Pajukoski, "On the waveforms for 5G mobile broadband communications,"in Proc. IEEE VTC-spring, pp. 1-5, May. 2015.

[3] S.S. Krishna Chaitanya Bulusu, H. Shaiek, and D. Roviras. "Prediction of spectral regrowth for FBMC-OQAM system using cumulants," in Proc. IEEE WiMob, pp. 402-406, Oct. 2014.

[4] D. Qu, S. Lu, and T. Jiang. "Multi-block joint optimization for the peakto- average power ratio reduction of FBMC-OQAM signals," IEEE Trans. Signal Process., vol. 61, no. 7, pp. 1605-1613, Jan. 2013.

[5] C. Ye, Z. Li, T. Jiang, C. Ni, and Q. Qi, "PAPR reduction of OQAMOFDM signals using segmental PTS scheme with low complexity," IEEE Trans. Broadcasting, vol. 60, no. 1, pp. 141-147, Mar. 2013.

[6] G. Cheng, H. Li, B. Dong, and S. Li, "An improved selective mapping method for PAPR reduction in OFDM/OQAM system," Communications and Network, vol. 5, no. 3, pp. 53-56, Sep. 2013.

[7] S. S. Krishna Chaitanya Bulusu, H. Shaiek, D. Roviras, and R. Zayani, "Reduction of PAPR for FBMC-OQAM systems using dispersive SLM technique," in Proc. ISWCS, pp. 26-29, Aug. 2014.

[8] A. Skrzypczak, J. P. Javaudin and P. Siohan, "Reduction of the Peak to average Power Ratio for OFDM-OQAM Modulation," in Proc. IEEE VTC-spring, pp. 2018-2022, May. 2006.

[9] Y. Zhou, T. Jiang, C. Huang, and S. Cui, "Peak-to-average power ratio reduction for OFDM/OQAM signals via alternativesignal method," IEEE Trans. Veh. Technol., vol. 63, no. 1, pp. 494-499, July. 2014.

[10] S. Lu, D. Qu, and Y. He, "Sliding window tone reservation technique for the peak-to-average power ratio reduction of FBMC-OQAM signals," IEEE Wireless Commun. Lett., vol. 1, no. 4, pp. 268-271, July. 2012.

[11] T. Ihalainen, A. Viholainen, T. Stitz, M. Renfors, and M. Bellanger, "Filter bank based multi-mode multiple access scheme for wireless uplink," in Proc. EUSIPCO, vol. 9, pp. 1354-1358, Aug. 2009.

[12] A. Viholainen, M. Bellanger, and M. Huchard, "PHYDAS-PHYsical layer for Dynamic AccesS and cognitive radio Report D5.1," Available: www.ict-phydyas.org/delivrables/PHYDYAS-D5-1.pdf, 2009.

[13] V. D. Neut et al., "PAPR reduction in FBMC systems using a smart gradient-projection active constellation extension method," in Proc. Telecommunication, 2014 21st Int. Conf. on, May 2014, pp. 134 - 139.

[14] C. Jose and S. M. Deepa, "Peak to Average Power Ratio Reduction and Inter Symbol Interference Cancellation of FBMCOQAM signals," International Journal of Engineering Research \& Technology, vol. 03, no. 03, pp. 1890 - 1894, Mar. 2014.

[15] G. Cheng, H. Li, B. Dong, and S. Li, “An Improved Selective Mapping Method for PAPR Reduction in OFDM/OQAM System," Scientific Research: Communications and Network, vol. 5, pp. 53-56, Sep. 2013.

[16] Z. He, J. Wang, X. Dy, J. Yan, and H. Xu, “A Novel PAPR Reduction Scheme in FBMC-OQAM Systems Based on Extend Candidate Transmit Sequences,” Journal of Information \& Computational Science 12:3 (2015), 5, pp. 915-925, Feb. 2015.

[17] Z. Kollar, L. Varga, B. Horvath, P. Bakki, and J. Bito, "Evaluation of Clipping Based Iterative PAPR Reduction Techniques for FBMC Systems," The Scientific World Journal, vol 2014, pp. 1 - 12, 2014.

[18] T. Jiang, C. Li, and C. Ni, "Effect of PAPR Reduction on Spectrum and Energy Efficiencies in OFDM Systems With ClassA HPA Over AWGN Channel," IEEE Trans. Broadcast., vol. 59, no. 3, pp. 513-519, Sept. 2013. 